\title{
Relationship between the angle of vertebral screws and spinal lateral angulation after fixation of thoracolumbar fractures via an anterior approach
}

\author{
L.T. Ma, Q. Gong, T. Li, Y.M. Song, F.X. Pei, X.D. Zhao, W.L. Zhang, \\ L.M. Liu, J.C. Zeng and H. Liu \\ Department of Orthopedics, West China Hospital, Sichuan University, \\ Chengdu, Sichuan, China \\ Corresponding author: H. Liu \\ E-mail: haoliu_12@sina.com
}

Genet. Mol. Res. 13 (4): 8135-8146 (2014)

Received July 29, 2013

Accepted March 25, 2014

Published October 7, 2014

DOI http://dx.doi.org/10.4238/2014.October.7.8

\begin{abstract}
This study investigated possible contributors to lateral spinal angulation after surgical fixation of thoracolumbar fractures via an anterior approach. We retrospectively examined lateral angulation in 172 cases of thoracolumbar fractures treated in this manner. The coronal Cobb angle and angles of the screws relative to the endplates were determined from radiographs. The patients completed the Short Form 36, Oswestry Disability Index, Japanese Orthopaedic Association Back Pain Evaluation Questionnaire, and Visual Analogue Scale at the final follow-up visit. The mean coronal Cobb angle was $0.75^{\circ} \pm 3.91^{\circ}$ $\left(-14.25^{\circ}\right.$ to $\left.14.55^{\circ}\right)$ preoperatively, $3.17^{\circ} \pm 4.07^{\circ}\left(-8.18^{\circ}\right.$ to $\left.14.01^{\circ}\right)$ immediately postoperatively, and $3.46^{\circ} \pm 4.21^{\circ}\left(-1.05^{\circ}\right.$ to $\left.17.27^{\circ}\right)$ at the final follow-up visit. The superior posterior and inferior anterior screws were more parallel to their respective endplates when the approach was made $\geq 2 v s \leq 1$ vertebral levels above the fracture $(\mathrm{P}<0.001)$. Lateral angulation was more likely when the approach was made $\leq 1 \quad v s \geq 2$ levels above the fracture $(\mathrm{P}<0.001)$. The coronal Cobb angle differed
\end{abstract}


significantly $(\mathrm{P}<0.01)$ between patients with lumbar and thoracic fractures. The immediate postoperative coronal Cobb angle correlated tightly with the sum of the screw angles (superior plus inferior posterior and/or inferior plus superior anterior). Lateral angulation may occur after surgical fixation of thoracic and lumbar fractures via an anterior approach. Non-parallelism between the vertebral screws and their corresponding endplates may predict postoperative lateral spinal angulation. Postoperative lateral angulation does not correlate with low back pain, quality of life, or preoperative lateral angulation.

Key words: Thoracic vertebrae; Lumbar vertebrae; Lateral angulation; Anterior approach; Thoracolumbar fracture

\section{INTRODUCTION}

Approximately $90 \%$ of spinal fractures occur at the thoracolumbar junction, and burst fractures constitute 10-20\% of such injuries (Kraemer et al., 1996). Although "unstable" thoracolumbar injuries are a common type of spinal trauma (Denis, 1983; Esses et al., 1990), their optimal treatment remains controversial, as evidenced by the variety of surgical management options available. These include posterior (Müller et al., 1999), anterior (Harrington et al., 1993; Magerl et al., 1994; Criscitiello and Frederickson, 1997; Ghanayem and Zdeblick, 1997; Kaneda et al., 1997; Parker et al., 2000; Alanay et al., 2001), and combined anteroposterior approaches (Denis, 1983; Sasso and Cotler, 1993). Many of the bone fragments that compress the spinal canal originate from the anterior vertebral body. Therefore, an anterior approach to the fixation of unstable thoracolumbar fractures has many advantages, including direct anterior decompression with little interference with the spinal cord and nerves in the canal as well as greater postoperative improvement in neurological function, more predictable solid anterior fusion, and a low rate of "re-kyphosis" (Harrington et al., 1993; Criscitiello and Frederickson, 1997). The aims of anterior surgery for the management of thoracolumbar burst fractures are to provide more direct decompression of the neural elements and more reliable stabilization of the spine (Hitchon et al., 1998). Screws placed into vertebrae should ideally be oriented parallel to the endplates (Denis, 1983; Esses et al., 1990). However, screws may be placed obliquely rather than parallel to the endplates, especially when attempting screw placement into L1, which is hindered by the superior margin of the wound. Previous studies have reported incidents of postoperative lateral angulation that was not present preoperatively (Sasso and Cotler, 1993; Wood et al., 2005; Yang et al., 2009). The purpose of this study was to investigate the possible main contributors to lateral angulation after surgical fixation of thoracolumbar burst fractures via an anterior approach in order to provide evidence to help surgeons improve their methods for anterior treatment of unstable thoracolumbar trauma.

\section{MATERIAL AND METHODS}

\section{Study population}

A total of 389 consecutive patients were treated for thoracolumbar fractures at West China Hospital between May 2004 and January 2008. Of these, 183 patients underwent an- 
terior surgical stabilization. The 172 patients for whom complete data were available were enrolled in this retrospective study. These cases included 124 males and 48 females aged $15-70$ years (mean, $39.09 \pm 11.68$ years). The causes of injury were falling in 118 patients, traffic accidents in 28 , impact from heavy material in 21 , jolting in 1 , and tumbling in 4 . The fracture site was T11 in 1 patient, T12 in 37, L1 in 88, L2 in 30, L3 in 15, and L4 in 3 . There were 2 cases in which fractures of two neighboring vertebras were operated simultaneously. The Frankel assessment of neurological status grade was A in 19 cases, B in 24, C in 45, D1 in $11, \mathrm{D} 2$ in 14, D3 in 28, and $\mathrm{E}$ in 31 . The time from injury to admission was 2 hours to 30 days (median, 8 days) and the time from admission to operation was 2-30 days (median, 8 days).

All patients were included based on written informed consent. This study was approved by the institutional review board and the ethics committee of our institution.

\section{Approach level}

The surgical approach was through the 9th rib in 5 cases, including 1 fracture of T11 and 4 of T12; through the 10th rib in 31 cases, including 29 fractures of T12, 1 of L1, and 1 of T12 and L1; through the 11th rib in 10 cases, including 2 fractures of T12, 7 of L1, and 1 of L2; through the 12th rib in 99 cases, including 1 fracture of T12, 75 of L1, 20 of L2, 2 of L3, and 1 of L1 and L2; through the 12th rib (with the 11th rib cut as well) in 2 cases of L1 vertebral fractures; and below the level of the ribs in 24 cases, including 2 fractures of L1, 8 of L2, 12 of L3, and 3 of L4 (Table 1).

\section{Table 1. Distribution of the approach level with respect to the vertebra fractured.}

\begin{tabular}{|c|c|c|c|c|c|c|c|}
\hline Approach level & Cases $(\mathrm{N})$ & T11 & $\mathrm{T} 12$ & L1 & L2 & L3 & L4 \\
\hline 9 & 5 & 1 & 4 & - & - & - & - \\
\hline 10 & $31^{*}$ & - & 30 & 2 & - & - & - \\
\hline 11 & 10 & - & 2 & 7 & 1 & - & - \\
\hline 12 & $99^{*}$ & - & 1 & 75 & 21 & 3 & - \\
\hline 11,12 & 2 & - & - & 2 & - & - & - \\
\hline Below ribs & 25 & - & - & 2 & 8 & 12 & 3 \\
\hline Total & 172 & 1 & 37 & 88 & 30 & 15 & 3 \\
\hline
\end{tabular}

*One with both T12 and L1 fractures and another with both L1 and L2 fractures.

\section{Surgical technique}

Care was taken to ensure that the patient was positioned in a true right lateral position in order to judge the anatomical structure. The anterior approach was usually made from the patient's left side to avoid damage to the inferior vena cava. Four types of incision were used, the transthoracic approach, transthoracic retroperitoneal approach, extrapleural retroperitoneal approach, and retroperitoneal approach; the type in each case depended on which vertebral segment was fractured. Care was taken to protect the peritoneum, abdominal viscera, and great vessels in the abdominal and thoracic cavities throughout the surgery. Lateral segmental vessels at above and below the level of the injury were ligated or coagulated proximal to the foramen. Sufficient dissection was performed to expose the entire lateral aspects of the vertebral body in fixation and fusion level. The vertebra fractured and the disc above and below were partially or sub-totally excised. The spinal canal was decompressed carefully. A screw was inserted appropriately into the vertebral body targeted for fixation. Then, the fractural vertebral 
body was excised, corrected, and strut grafted. A linked rod or plate was placed, and all parts of the internal fixation system were locked. The position of the internal fixation system was verified intraoperatively. Anterior fixation was performed with the Z-plate (Medtronic, Sofamor Danek) in 63 patients, the Antares (Medtronic, Sofamor Danek) in 59 patients, and the Profile (Depuy AcroMed, Johnson \& Johnson Company) in 50 patients. The vertebra fractured was reconstructed with a titanium-mesh cage packed with local autograft (87 patients), structural iliac autograft (79 patients), structural rib and local autograft (4 patients), or allograft bone ( 2 patients). Single-level anterior fixation was performed in 15 patients, 2-level fixation in 155 patients, and 3-level fixation in 2 patients.

\section{Radiographic evaluation}

Postoperatively, posterior and lateral plain radiographs were taken of the spinal segments where the internal fixation system had been placed. The 4 screws of the internal fixation system were designated, in the cephalic to caudal direction, the superior posterior screw (SP), inferior anterior screw (IA), superior anterior screw (SA), and inferior posterior screw (IP). The angles between the superior endplate and the SP and IA were designated angles SP and IA, and the angles between the inferior endplate and the SA and IP were designated angles SA and IP. The Cobb angle was the angle between a line extending from the superior endplate of the superior vertebra in which the screws were located and a line extending from the inferior endplate of the inferior vertebra in which the screws were located (not the superior and inferior fractured vertebral bodies) (Gelb et al., 1995). The coronal Cobb angle was measured on radiographs taken preoperatively, immediately postoperatively, and at the final follow-up visit. And the angle SP, angle IA, angle SA, and angle IP were measured on the immediate postoperative radiographs. The values of all of these angles were defined as positive when the angle opened in the direction of the internal fixation system and negative when they opened in the opposite direction (Figure 1). All of the angles were measured 3 times by an independent investigator, and the final result was the mean of the 3 measurements.

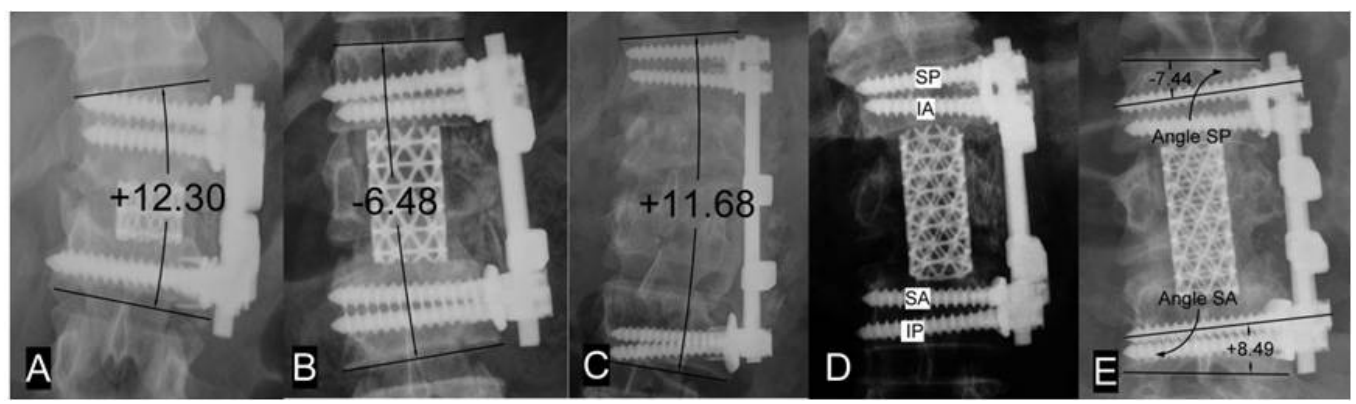

Figure 1. Schematic diagram of the locations of the superior posterior (SP), inferior anterior (IA), superior anterior (SA), and inferior posterior (IP) screws and examples of positive and negative values of angles SP, IA, SA, and IP and the coronal Cobb angle on posteroanterior radiographs. A. Positive coronal Cobb angle after single-segment fixation via an anterior approach. B. Negative coronal Cobb angle after conventional 2-segment fixation via an anterior approach. C. Positive coronal Cobb angle after 3-segment fixation via an anterior approach. D. The screws are designated SP, IA, SA, and IP in cephalic to caudal order. E. The angles between the superior endplate and screws SP and IA are designated angles SP and IA, and the angles between the inferior endplate and screws SA and IP are designated angles SA and IP. The angle is defined as positive when it opens towards the internal fixation system and negative when it opens in the opposite direction. 


\section{Contents of interview}

The primary outcome parameters were residual pain and functional outcome. At the final follow-up visit, the Visual Analogue Scale (VAS) was administered to assess residual pain $(0=$ no pain, $10=$ unbearable pain $)$, and the Oswestry Disability Index (ODI) and Japanese Orthopaedic Association Back Pain Evaluation Questionnaire (JOABPEQ) scores were calculated. The Short Form 36 Health Survey (SF-36) was used to evaluate each patient's physical and mental status. Neurologic status was assessed preoperatively and postoperatively according to the modified system of Frankel.

\section{Statistical analysis}

Statistical analyses were performed using SPSS 13.0 (SPSS, Inc., Chicago, IL, USA). The preoperative, immediate postoperative, and final follow-up coronal Cobb angles, the SP, IP, and SP + IP angles, and the postoperative coronal Cobb angle were compared using oneway analysis of variance (ANOVA). The results of the VAS, JOABPEQ, ODI, and 8 dimensions of the SF-36 were compared among the 3 immediate postoperative Cobb angle groups. In addition, the relationship between the immediate postoperative coronal Cobb angle and angle SP + IP (and/or angle IA + SA) was examined by linear correlation analysis. A P value of less than 0.05 was considered to be statistically significant.

\section{RESULTS}

The 172 cases were followed up for 6-49 months (mean, 39 months). The surgical time (from initial incision to application of the dressing) was $220 \pm 46 \mathrm{~min}$ (range, 130-400 $\mathrm{min}$ ). One incision developed superficial infection that was noted 7 days postoperatively; it was debrided once and healed well, and all other incisions healed by first intention. Solid fusion of the upper and lower surfaces of the bone graft to the vertebral bodies was noted at the time of the final follow-up visit in all cases, including 2 patients in which one screw migrated but did not become free. No pseudarthrosis was noted in any case.

\section{Radiologic measurements}

The patients were divided into 3 groups on the basis of the coronal Cobb angle immediately after surgery: $0-5^{\circ}, 5-10^{\circ}$, or $>10^{\circ}$. The mean coronal Cobb angle was $0.75^{\circ} \pm 3.91^{\circ}$ (range, $-14.25^{\circ}$ to $14.55^{\circ}$ ) preoperatively, $3.17^{\circ} \pm 4.07^{\circ}$ (range, $-8.18^{\circ}$ to $14.01^{\circ}$ ) immediately postoperatively, and $3.46^{\circ} \pm 4.21^{\circ}\left(-1.05^{\circ}\right.$ to $\left.17.27^{\circ}\right)$ at the final follow-up visit. The coronal Cobb angle increased significantly after surgery $(\mathrm{P}<0.001)$. The lateral angulation did not differ significantly between the immediate postoperative and final follow-up measurements $(\mathrm{P}>0.05)$.

\section{Incision level}

The patients were divided into 2 groups according to the number of vertebral segments between level of the incision and that of the fractured vertebral body: $\geq 2$, such as removal of the L1 body through the 11th rib, and $\leq 1$, such as removal of the L1 body through the 
12th rib. As a general rule, when using an anterior approach to treat a thoracolumbar fracture the incision is made through the 2 vertebral segments above the vertebra fractured, such as removal of the L1 body through the 11th or even the 10th rib. However, the incision may instead be made through the 1 vertebral segment above the fracture due to error, habit, or a desire to avoid damaging the pleura. We compared the preoperative, immediate postoperative, and coronal Cobb angles with respect to the number of segments between the fracture and the incision (Figure 2) and found that the immediate postoperative and follow-up coronal Cobb angles differed significantly between the incision level $\geq 2$ and incision level $\leq 1$ groups $(\mathrm{P}<$ $0.001)$ despite little difference in their preoperative coronal Cobb angles $(\mathrm{P}>0.05)$. Making the incision too low leads to insufficient exposure, which may result in failure to insert the screws parallel to their corresponding endplates. As shown in Figure 3, angles SP and IA but not angle SA or IP differed between the incision level $\geq 2$ and incision level $\leq 1$ groups $(\mathrm{P}<$ 0.001 and $\mathrm{P}>0.05$, respectively).

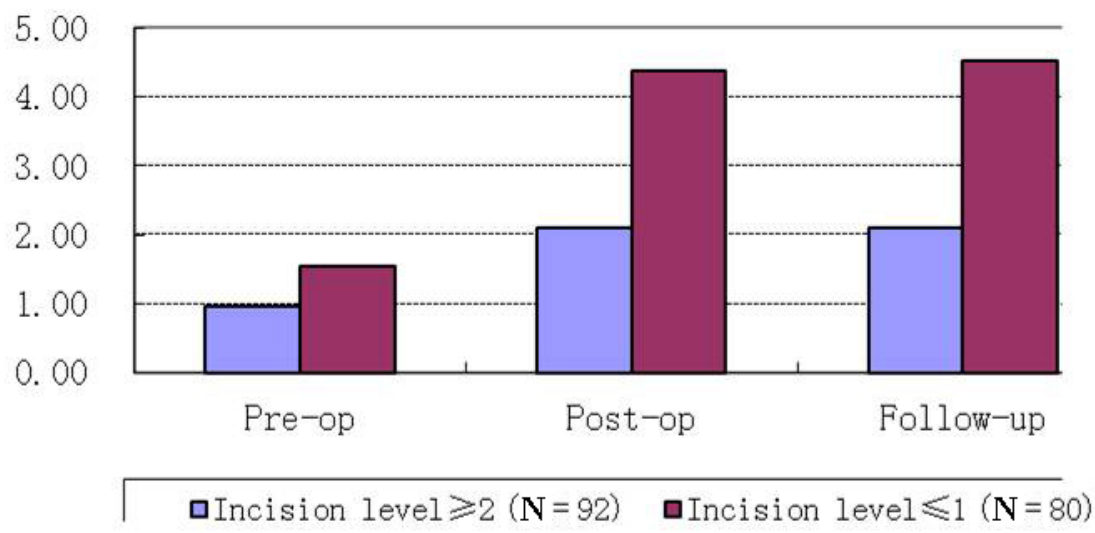

Figure 2. Coronal Cobb angle with respect to the incision level (angle). Pre-op $=$ preoperative; post-op $=$ immediately postoperative; follow-up = at the time of the final follow-up visit.

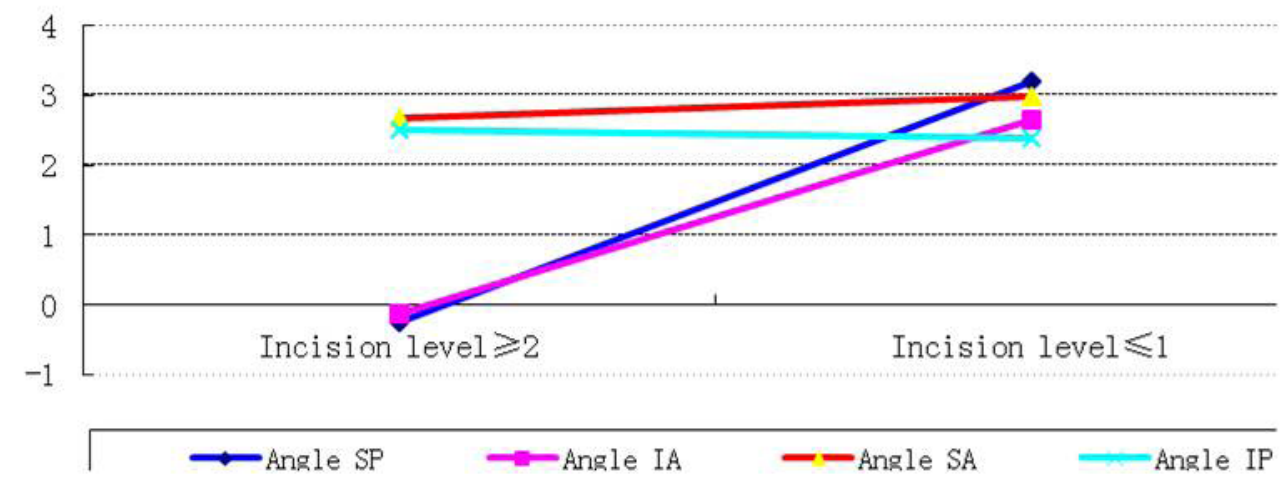

Figure 3. Superior posterior (SP), inferior anterior (IA), superior anterior (SA), and inferior posterior (IP) screw angles with respect to the incision level. 


\section{Thoracic or lumbar spine}

The patients comprised 41 with thoracic vertebral fractures and 137 with lumbar vertebral fractures. Comparison of the preoperative, immediate postoperative, and follow-up coronal Cobb angles between the thoracic and lumbar fractures (Figure 4) showed no significant difference in the preoperative value $(\mathrm{P}>0.05)$ but significant differences in the immediate postoperative and follow-up values $(\mathrm{P}<0.05)$.

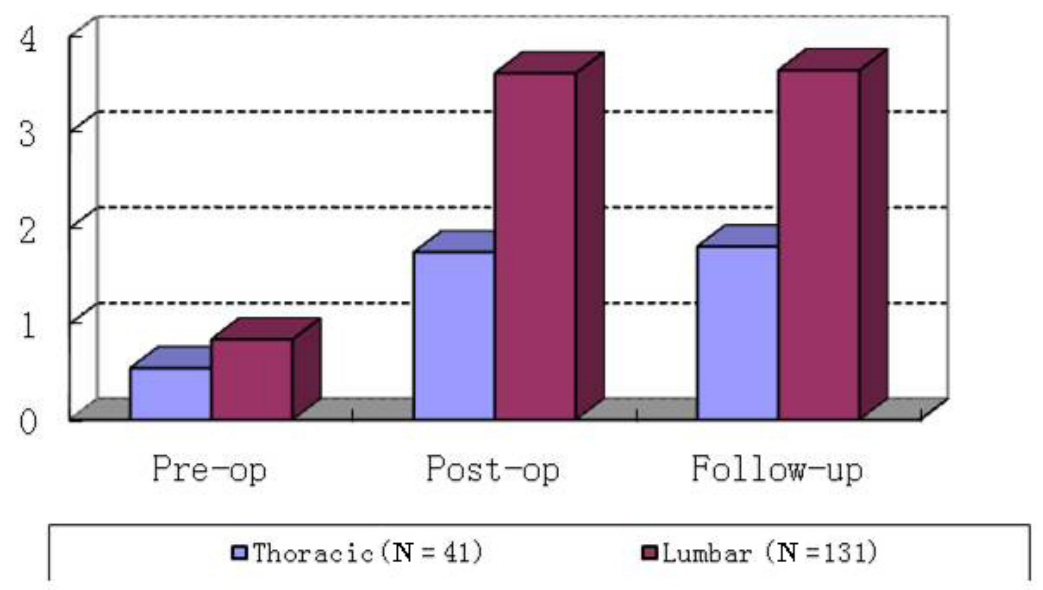

Figure 4. Coronal Cobb angle with respect to the location of the fracture in the thoracic versus lumbar spine. Pre-op $=$ preoperative; post-op $=$ immediately postoperative; follow-up $=$ at the time of the final follow-up visit.

\section{Angle SP + IP (and/or SA + IA)}

Table 2 shows the comparison between angle SP + IP and the immediate postoperative coronal Cobb angle. The immediate postoperative coronal Cobb angle differed significantly $(\mathrm{P}<0.05)$ from angle $\mathrm{SP}+\mathrm{IP}$ of screw insertion only in the $0-5^{\circ}$ group $\left(\mathrm{P}>0.05\right.$ for the $5-10^{\circ}$ and $>10^{\circ}$ groups). As IA and SA in the Antares system might produce the same effect on lateral angulation in the Antares system, whereas IA and SA are only accessory screws in the Z-plate and Profile systems and are absent in the Mono-screw-rod system, we compared screw insertion angle IA + SA with the immediate postoperative coronal Cobb angle in the $5-10^{\circ}$ groups and found no significant difference $(\mathrm{P}>0.05)$. The $>10^{\circ}$ group included only 3 cases in which the Antares system was used; therefore, no statistical analysis was performed. However, the numbers appeared similar: angle IA + SA and the immediate postoperative coronal Cobb angle were $14.15^{\circ}$ and $14.01^{\circ}, 9.73^{\circ}$ and $10.41^{\circ}$, and $13.50^{\circ}$ and $12.14^{\circ}$, respectively. Table 3 shows the analysis of the linear correlation between the immediate postoperative coronal Cobb angle and angle SP + IP in each group. The immediate postoperative coronal Cobb angle correlated tightly with the screw insertion angle SP + IP in every group. As described above, we calculated the degree of linear correlation between the postoperative coronal Cobb angle and angle $\mathrm{IA}+\mathrm{SA}$ in the Antares system subgroups and found no correlation in the $0-5^{\circ}$ group but a significant correlation in the $5-10^{\circ}$ group. The angles also appeared similar in the $>10^{\circ}$ group. 
Table 2. Comparison between the sum of the immediate postoperative superior and inferior posterior screw angles (SP + IP) and coronal Cobb angle in each Cobb angle group $\left({ }^{\circ}\right.$, means \pm standard deviation).

\begin{tabular}{lccccc}
\hline Group & Angle SP & Angle IP & Angle SP + IP $(\mathrm{X})$ & Cobb angle $(\mathrm{Y})$ & $\mathrm{P}(\mathrm{X}$ versus Y) \\
\hline $0-5^{\circ}(\mathrm{N}=109)$ & $0.50 \pm 3.95$ & $1.88 \pm 3.22$ & $2.36 \pm 3.74$ & $1.45 \pm 2.43$ & 0.037 \\
$5-10^{\circ}(\mathrm{N}=54)$ & $2.24 \pm 4.44$ & $3.13 \pm 4.35$ & $5.36 \pm 6.19$ & $5.10 \pm 4.35$ & 0.796 \\
$>10^{\circ}(\mathrm{N}=9)$ & $6.47 \pm 3.35$ & $5.09 \pm 4.49$ & $12.77 \pm 1.65$ & $11.71 \pm 1.29$ & 0.149 \\
\hline
\end{tabular}

$\mathrm{SP}=$ superior posterior; IP $=$ inferior posterior; $\mathrm{IA}=$ inferior anterior; $\mathrm{SA}=$ superior anterior.

Table 3. Linear correlation between the post-operative coronal Cobb angle in each group (and Antares subgroup) and the sum of the superior and inferior posterior $(\mathrm{SP}+\mathrm{IP})$ [and/or inferior and superior anterior (IA + SA)] angles.

\begin{tabular}{lcccc}
\hline Group & Coefficient B & Standardized coefficient & $t$ & P \\
\hline $0-5^{\circ}(\mathrm{N}=109)$ & 0.315 & 0.486 & 5.745 & 0.000 \\
$5-10^{\circ}(\mathrm{N}=54)$ & 0.552 & 0.743 & 8.002 & 0.000 \\
$>10^{\circ}(\mathrm{N}=9)$ & 0.627 & 0.728 & 2.813 & 0.026 \\
\hline
\end{tabular}

\section{ODI, JOABPEQ, and VAS}

The Oswestry Disability Index has 10 specific categories: pain, personal care, lifting, walking, sitting, standing, sleeping score, sexual activity, social activities, and traveling. The maximum score is $100(0-20 \%$ indicates minimal disability, $20-40 \%$ moderate disability, $60-$ $80 \%$ significant disability, and $>80 \%$ either complete disability or exaggeration of symptoms). The median overall Oswestry Disability Index score was 24\% (range, 2-78\%). At the final follow-up visit the median VAS score was 2 (range, 0-6) and the median JOABPEQ score 24 (1030 ). Figure 5 shows a comparison of the ODI, VAS, and JOABPEQ scores among the 3 lateral angulation groups $\left(0-5^{\circ}, 5-10^{\circ}\right.$, and $\left.>10^{\circ}\right)$. There was no significant difference in the ODI, VAS, or JOABPED score among the $0-5^{\circ}, 5-10^{\circ}$, and $>10^{\circ}$ groups $(\mathrm{P}>0.05)$. The SF-36 comprises 8 domains: physical functioning $(\mathrm{PF})$, role limitations due to physical, health (RP), bodily pain (BP), general health perceptions (GH), vitality (VT), social functioning (SF), role limitations due to emotional problems (RE), and general mental health (MH). Two summary measures of physical (PCS) and mental (MCS) health are constructed from the 8 scales. The patients completed the SF-36 survey at the last follow-up visit. To investigate whether the lateral spinal angulation influenced the patients' quality of life, we compared the 8 components of the SF-36 among the $0-5^{\circ}, 5-10^{\circ}$, and $>10^{\circ}$ groups (Table 4$)$ and found no significant difference $(\mathrm{P}>0.05)$.

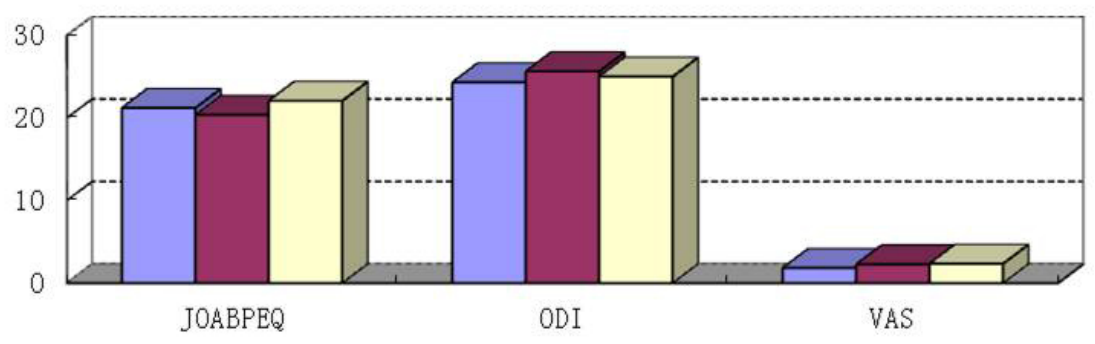

$$
\text { 口0-5 } 5^{\circ} \operatorname{group}(\mathrm{N}=109) \quad \square 5^{\circ}-10^{\circ} \quad \operatorname{group}(\mathrm{N}=54) \quad \text { a0ver } 10^{\circ} \quad \text { group }(\mathrm{N}=9)
$$

Figure 5. Functional outcome with respect to the immediate postoperative coronal Cobb angle $\left({ }^{\circ}\right.$, means \pm standard deviation). 


\begin{tabular}{|c|c|c|c|}
\hline Dimension & $0-5^{\circ}$ group & $5-10^{\circ}$ group & $>10^{\circ}$ group \\
\hline $\mathrm{PF}$ & $73.33 \pm 23.86$ & $70.37 \pm 21.50$ & $71.07 \pm 22.30$ \\
\hline $\mathrm{RP}$ & $65.00 \pm 32.17$ & $59.76 \pm 30.29$ & $61.69 \pm 28.74$ \\
\hline $\mathrm{BP}$ & $72.22 \pm 22.98$ & $65.91 \pm 20.99$ & $63.06 \pm 21.46$ \\
\hline GH & $77.00 \pm 14.18$ & $65.00 \pm 12.40$ & $66.32 \pm 14.03$ \\
\hline VT & $76.88 \pm 22.45$ & $68.75 \pm 18.67$ & $67.31 \pm 19.25$ \\
\hline SF & $75.00 \pm 27.00$ & $78.33 \pm 30.42$ & $70.42 \pm 28.79$ \\
\hline RE & $70.00 \pm 26.41$ & $67.79 \pm 30.14$ & $65.82 \pm 29.07$ \\
\hline $\mathrm{MH}$ & $75.00 \pm 20.55$ & $69.26 \pm 20.02$ & $67.07 \pm 20.66$ \\
\hline
\end{tabular}

$\mathrm{PF}=$ physical functioning; $\mathrm{RP}=$ role limitations due to physical health; $\mathrm{BP}=$ bodily pain; $\mathrm{GH}=$ general health perceptions; $\mathrm{VT}=$ vitality; $\mathrm{SF}=$ social functioning; $\mathrm{RE}=$ role limitations due to emotional problems; $\mathrm{MH}=$ general mental health.

\section{Neurological recovery}

None of the patients exhibited neurological deterioration after anterior decompression and stabilization. All patients except those with a preoperative Frankel grade of E exhibited various degrees of recovery of neurological function at the final follow-up visit. The neurological function had improved by at least 1 grade in 138 of the 172 patients $(80.2 \%$; Table 5).

\begin{tabular}{|c|c|c|c|c|c|c|c|c|}
\hline \multirow[t]{2}{*}{ Preoperative frankel grade } & \multirow[t]{2}{*}{ Cases } & \multicolumn{7}{|c|}{ Frankel grade at the last follow-up visit } \\
\hline & & $\mathrm{A}$ & $\mathrm{B}$ & $\mathrm{C}$ & D1 & $\mathrm{D} 2$ & D3 & $\mathrm{E}$ \\
\hline A & 19 & 14 & 3 & 2 & & & & \\
\hline B & 24 & & 12 & 7 & 2 & 1 & 2 & \\
\hline $\mathrm{C}$ & 45 & & & 8 & 12 & 7 & 13 & 5 \\
\hline D1 & 11 & & & & & & 9 & 2 \\
\hline D2 & 14 & & & & & & & 14 \\
\hline D3 & 28 & & & & & & & 28 \\
\hline $\mathrm{E}$ & 31 & & & & & & & 31 \\
\hline Total & 172 & 14 & 15 & 17 & 14 & 8 & 24 & 80 \\
\hline
\end{tabular}

\section{DISCUSSION}

The anterior approach to the thoracolumbar spine was first developed by Hodgson and Stock, who advocated it primarily for the drainage of tuberculous abscesses (Sasso et al., 2005). The retroperitoneal approach to the thoracolumbar spine for the decompression of late, malunited burst fractures, usually those associated with kyphotic deformity and canal encroachment, was developed by Bohlman (Fontijne et al., 1992). An anterior device designed by Dunn yielded excellent initial results for reduction and fixation of thoracolumbar burst fractures; however, vascular complications led to the discontinuation of this device. Kaneda developed an anterior fixation device that uses vertebral body staples and screws connected by 2 cross-linked threaded longitudinal rods (Kaneda et al., 1984). The Kaneda device represented a revolution in the development of anterior instrumentation systems for the thoracolumbar spine, and such complications as nonunion, device failure, loss of reduction, and vascular or neurologic damage have decreased or disappeared since its introduction. Current 
systems, such as the $\mathrm{Z}$ plate anterior thoracolumbar fixation system, University Plate, Anterior Thoracolumbar Locking Plate, Texas Scottish Rite Hospital device (TSRH), Profile, and Antares sought to add to these achievements by decreasing the profile of the implants, improving their biomechanical strength (in response to other device-related failures), and using titanium rather than stainless steel to improve postoperative imaging properties. The West China Hospital has used an anterior approach to treat thoracolumbar fractures since 1984 (Kraemer et al., 1996). The indications for anterior approach surgery include secondary neurologic deficits, anterior canal compromise of $>50 \%$, kyphotic deformity of $>30^{\circ},>50 \%$ loss of anterior vertebral height, progressive kyphosis, or late pain secondary to malunion of the burst fracture. As indicated previously, the anterior approach has more benefits than the posterior approach, and these advantages are especially obvious in cases of complicated fractures. However, the anterior approach has some drawbacks, including greater blood loss and risk for severe injury to the patient. Postoperative lateral angulation is another such shortcoming. In the present study, 63 of 172 patients (36.6\%) exhibited lateral angulation of $>5^{\circ}$. Spinal fixation via an anterior approach exposes only one side of the spine, and the internal fixation system is attached to that side alone; it is thus a form of partial fixation. Lateral angulation may develop due to improper orientation of the screws, excessive strut, suboptimal location of the implant, or failure to reverse the titanium mesh and the waist bridge removed in time. Ghanayem and Zdeblick (1997) and McDonough et al. (2004) advocate reflexing the operating table during the anterior approach to the thoracolumbar spine in order to facilitate exposure (like the waist bridge), but this must be reversed before instrumentation. Four types of incision, transthoracic, transthoracic retroperitoneal, extrapleural retroperitoneal, and retroperitoneal, are possible depending on which vertebral segment is fractured. The dissection then proceeds along the rib 1 or 2 levels above the injured segment(s) (Sasso and Cotler, 1993). The thoracolumbar junction is usually approached through the site of the 10th or 11th rib, which may require partial division of the diaphragm. A 12th rib approach should be used if insertion of screws into L1 is planned (Esses et al., 1990); this is performed by dividing the diaphragm between stay sutures 1 to $2 \mathrm{~cm}$ from the chest wall. The diaphragm is incised and reflected for most L1 fractures. The retroperitoneal approach can be used to place fixation screws as high as L2. Fractures of L2 and L3 are managed using a subdiaphragmatic retroperitoneal approach (Sasso et al., 2005). Attempting screw placement into L1 is hindered by the superior margin of the wound and may result in screws being placed obliquely rather than parallel to the endplates. Failure to select the proper level from which to approach and fix the spine is an avoidable technical error.

This study compared the preoperative, immediate postoperative, and follow-up coronal Cobb angles between patients whose the level of the incision was $\geq 2$ vertebrae above the fracture and those in whom it was $\leq 1$ level above. The postoperative lateral angulation appeared greater in the incision level $\leq 1$ than in the incision level $\geq 2$ patients. The postoperative angles SP and IA were also greater in the incision level $\leq 1$ group than in the incision level $\geq 2$ group. However, angles SA and IP did not differ significantly between the 2 groups. Making the approach too low results in insufficient exposure, which may lead to failure to insert the screws parallel to the corresponding endplate. In 2 cases in this study, an anterior approach to an L1 vertebral body fracture was made through the 12th rib and the 11th rib was transected as well to ensure placement of the proximal screws, SP and IA, almost parallel to the endplate; this appears to be a sound practice. The present study suggests that postoperative lateral angulation is more likely in patients in whom the incision was made $\leq 1$ level above the fracture, indicating that the level of the incision plane directly or indirectly affects 
the postoperative coronal deformity of the spine. Lateral angulation was also more frequent after fixation of fractures of the lumbar spine. The lumbar spine is quite flexible. Fixation of lumbar vertebral fractures, particularly via an anterior approach with resection of the 12th rib, is usually hindered by the upper edge of the incision, which may prevent placement of the screws parallel to the endplate. As the screw nut at the end of the nail is tightened, the angle between the screw and the plate will gradually approach $90^{\circ}$ passively, resulting in vertebral tilt. The thoracic spine has a more limited range of motion and does not easily tilt noticeably; the energy may instead dissipate by damaging the bone. The screws should be placed parallel to the corresponding endplate (Schnee and Ansell, 1997). This study showed that non-parallel screw placement may lead to spinal lateral angulation. Most internal fixation systems, such as the Antares, Profile, and Mono-screw-rod systems, have a strict requirement for a connection angle of $90^{\circ}$. This requires that the screws parallel the endplates, i.e., angle SP + IP is equal to $0^{\circ}$. If angle $\mathrm{SP}+\mathrm{IP}$ is positive, both the SP and IP screws will force the vertebral body to tilt when the screw nut at the end of the nail is tightened, resulting in a positive postoperative Cobb angle. Conversely, when angle SP + IP is negative, the postoperative Cobb angle will also be negative. This means that both positive and negative deviation of the SP + IP angle result in lateral angulation. In contrast, if the angle of 1 screw is positive and the other is negative, the resulting angle SP + IP is small and lateral angulation may not develop. Notably, the SP and IP screws of the Z-plate can be allowed $24^{\circ}$ of rotation, which could explain the lack of correlation between the immediate postoperative Cobb angle and angle SP + IP in the $0-5^{\circ}$ group. Correlation indeed was detected in $5-10^{\circ}$ and $10-15^{\circ}$ groups perhaps because the $24^{\circ}$ rotation did not work.

After follow-up, the patients in this study scored 2-78\% (median, 24\%) on the Oswestry dysfunction scale. This result is consistent with the Oswestry score of $21 \%$ in Wood's report of 20 cases of thoracolumbar burst fractures treated via an anterior surgical approach (Wood et al., 2005). Furthermore, Boucher reported a mean Oswestry score of 25\% (standard deviation, 22.59\%) 3.2 years after surgical treatment of lumbar burst fractures (Boucher et al., 2001). The median VAS pain score in the present study was 2 points; this pain could be due to injury to the spinal cord, cauda equina, and/or lower abdominal cutaneous nerves. We found no significant difference in the JOABPEQ score, Oswestry score, or VAS score with respect to the postoperative $\mathrm{Cobb}$ angle, indicating that the magnitude of the postoperative coronal $\mathrm{Cobb}$ angle had no significant effect on low back pain in our patients. The study also compared the 8 domains of the SF-36 among the Cobb angle groups and found no significant difference.

In conclusion, postoperative lateral angulation had no significant effect on quality of life; therefore, surgical correction is unnecessary. We hope that this result will help clinicians to assuage their patients' worries concerning postoperative lateral angulation. However, we can do more to avoid inappropriate screw angles.

\section{Conflicts of interest}

The authors declare no conflict of interest.

\section{REFERENCES}

Alanay A, Acaroglu E, Yazici M, Oznur A, et al. (2001). Short-segment pedicle instrumentation of thoracolumbar burst fractures: does transpedicular intracorporeal grafting prevent early failure? Spine 26: 213-217. 
Boucher M, Bhandari M and Kwok D (2001). Health-related quality of life after short segment instrumentation of lumbar burst fractures. J. Spinal Disord. 14: 417-426.

Criscitiello AA and Fredrickson BE (1997). Thoracolumbar spine injuries. Orthopedics 20: 939-944.

Denis F (1983). The three column spine and its significance in the classification of acute thoracolumbar spinal injuries. Spine 8: 817-831.

Esses SI, Botsford DJ and Kostuik JP (1990). Evaluation of surgical treatment for burst fractures. Spine 15: 667-673.

Fontijne WP, de Klerk LW, Braakman R, Stijnen T, et al. (1992). CT scan prediction of neurological deficit in thoracolumbar burst fractures. J. Bone Joint Surg. Br. 74: 683-685.

Gelb DE, Lenke LG, Bridwell KH, Blanke K, et al. (1995). An analysis of sagittal spinal alignment in 100 asymptomatic middle and older aged volunteers. Spine 20: 1351-1358.

Ghanayem AJ and Zdeblick TA (1997). Anterior instrumentation in the management of thoracolumbar burst fractures. Clin. Orthop. Relat. Res. 89-100.

Harrington RM, Budorick T, Hoyt J, Anderson PA, et al. (1993). Biomechanics of indirect reduction of bone retropulsed into the spinal canal in vertebral fracture. Spine 18: 692-699.

Hitchon PW, Torner JC, Haddad SF and Follett KA (1998). Management options in thoracolumbar burst fractures. Surg. Neurol. 49: 619-626.

Kaneda K, Abumi K and Fujiya M (1984). Burst fractures with neurologic deficits of the thoracolumbar-lumbar spine. Results of anterior decompression and stabilization with anterior instrumentation. Spine 9: 788-795.

Kaneda K, Taneichi H, Abumi K, Hashimoto T, et al. (1997). Anterior decompression and stabilization with the Kaneda device for thoracolumbar burst fractures associated with neurological deficits. J. Bone Joint Surg. Am. 79: 69-83.

Kraemer WJ, Schemitsch EH, Lever J, McBroom RJ, et al. (1996). Functional outcome of thoracolumbar burst fractures without neurological deficit. J. Orthop. Trauma 10: 541-544.

Magerl F, Aebi M, Gertzbein SD, Harms J, et al. (1994). A comprehensive classification of thoracic and lumbar injuries. Eur. Spine J. 3: 184-201.

McDonough PW, Davis R, Tribus C and Zdeblick TA (2004). The management of acute thoracolumbar burst fractures with anterior corpectomy and Z-plate fixation. Spine 29: 1901-1908.

Müller U, Berlemann U, Sledge J and Schwarzenbach O (1999). Treatment of thoracolumbar burst fractures without neurologic deficit by indirect reduction and posterior instrumentation: bisegmental stabilization with monosegmental fusion. Eur. Spine J. 8: 284-289.

Parker JW, Lane JR, Karaikovic EE and Gaines RW (2000). Successful short-segment instrumentation and fusion for thoracolumbar spine fractures: a consecutive 41/2-year series. Spine 25: 1157-1170.

Sasso RC and Cotler HB (1993). Posterior instrumentation and fusion for unstable fractures and fracture-dislocations of the thoracic and lumbar spine. A comparative study of three fixation devices in 70 patients. Spine 18: 450-460.

Sasso RC, Best NM, Reilly TM and McGuire RA Jr (2005). Anterior-only stabilization of three-column thoracolumbar injuries. J. Spinal Disord. Tech. 18 (Suppl): S7-14.

Schnee CL and Ansell LV (1997). Selection criteria and outcome of operative approaches for thoracolumbar burst fractures with and without neurological deficit. J. Neurosurg. 86: 48-55.

Wood KB, Bohn D and Mehbod A (2005). Anterior versus posterior treatment of stable thoracolumbar burst fractures without neurologic deficit: a prospective, randomized study. J. Spinal Disord. Tech. 18 (Suppl): S15-S23.

Yang Z, Guo H, Gui S, Wang J, et al. (2009). Anterior surgery for fourth lumbar burst fractures. Zhongguo Xiu. Fu Chong. Jian. Wai Ke Za Zhi 23: 793-796. 